\title{
COMPARISON OF SOME FAMILIES OF REAL FUNCTIONS IN ALGEBRAIC TERMS
}

\author{
MaŁgorzata FilipcZaK — Gertruda Ivanova
}

\begin{abstract}
We compare families of functions related to the Darboux property (functions having the $\mathcal{A}$-Darboux property) with family of strong Świagtkowski functions using the notions of strong $\mathfrak{c}$-algebrability. We also compare families of functions associated with density topologies.
\end{abstract}

\section{Introduction}

We will work with real functions defined on the real line, so if we write "function", then we mean $f: \mathbb{R} \rightarrow \mathbb{R}$. Throught the whole paper, when open or closed sets are mentioned, we mean the Euclidean topology in $\mathbb{R}$. We denote by $\bar{A}(\operatorname{Int}(A))$ the closure (interior) of the set $A$.

Recall that a function $f$ is quasi-continuous at a point $x$ if for every neighbourhood $U$ of $x$ and for every neighbourhood $V$ of $f(x)$ there exists a nonempty open set $G \subset U$ such that $f(G) \subset V$. A function $f$ is quasi-continuous (briefly $f \in \mathcal{Q}$ ) if it is quasi-continuous at each point. The notion of quasi-continuity was introduced by S. Ke m p is t y in $1932[12$.

A function $f: \mathbb{R} \rightarrow \mathbb{R}$ is called a strong Swiatkowski function if for each interval $(a, b) \subset \mathbb{R}$ and for each $\lambda$ between $f(a)$ and $f(b)$ there exists a point $x \in(a, b)$ such that $f(x)=\lambda$ and $f$ is continuous at $x$ [17]. Hence, the family $\mathcal{D}_{s}$ of strong Świątkowski functions is situated somewhere in the road between the family $\mathcal{C}$ of all continuous functions and the family $\mathcal{D} \mathcal{Q}$ of all Darboux quasi-continuous functions.

The notion of strong Świątkowski property was introduced by A. Maliszewski in [17. He showed that $\mathcal{D}_{s}$ is dense in $\mathcal{D} \mathcal{Q}$, so $\mathcal{D}_{s}$ is "not so far" from $\mathcal{D} Q$ (for more information about this class, see [16]-[19]).

(c) 2017 Mathematical Institute, Slovak Academy of Sciences. 2010 Mathematics Subject Classification: 26A15, $54 \mathrm{C} 08$.

Keyw or ds: Darboux property, strong Świątkowski property, quasi-continuity, strong algebrability. 
On the other hand, J. W ód ka [27] proved that the set $\left(\mathcal{D} Q \backslash \mathcal{D}_{s}\right) \cup\{\Theta\}$ contains a $\mathfrak{c}$-generated algebra, i.e., $\mathcal{D} \mathcal{Q}$ is much larger than $\mathcal{D}_{s}$ if we compare these families in algebraic terms.

In this paper, we will generalize the result obtained by J. Wó d k a. We will compare, in algebraic terms, $\mathcal{D}_{s}$ and $\mathcal{D} \mathcal{Q}$ with the family $\mathcal{D}_{a p}$ of approximately Darboux functions and the family $\mathcal{D}_{\mathcal{J}-a p}$ of $\mathcal{J}_{\text {-approximately Darboux functions. }}$ We will use the exponential-like method introduced in [2].

Let $\mathcal{B} r$ denotes a family of all sets having the Baire property, and $n \cdot A$ stands for a set $\{n \cdot a: a \in A\}$. We will write $<a, b>$ instead of $(\min \{a, b\}, \max \{a, b\})$.

\section{2. $\mathcal{A}$-continuity and $\mathcal{A}$-Darboux property}

We start with the definition which helps us describe approximate continuity and J-approximate continuity as well as quasi-continuity in the same way.

Let $\mathcal{A} \subset \mathcal{P}(\mathbb{R})$, where $\mathcal{P}(\mathbb{R})$ is a power set of $\mathbb{R}$.

Definition 1 ([10]). We say that $f$ is $\mathcal{A}$-continuous at the point $x \in \mathbb{R}$ if for each open set $V \subset \mathbb{R}$ with $f(x) \in V$ there exists a set $A \in \mathcal{A}$ such that $x \in A$ and $f(A) \subset V$. We say that $f$ is $\mathcal{A}$-continuous $\left(f \in \mathcal{C}_{\mathcal{A}}\right)$ if $f$ is $\mathcal{A}$-continuous at each point $x \in \mathbb{R}$.

Remind that $x$ is a density point of a measurable set $A \subset \mathbb{R}$ when

$$
\lim _{h \rightarrow 0^{+}} \frac{m(A \cap[x-h, x+h])}{2 h}=1
$$

and the family of all measurable sets which any point is its density point is a topology called the density topology.

Now, let $\mathcal{J}$ be a $\sigma$-ideal of sets of the first category. We will say that a property holds J-almost everywhere (briefly J-a.e.) if the set of all points which do not have this property belongs to $\mathcal{J}$.

We will say that a sequence $\left\{f_{n}\right\}_{n \in \mathbb{N}}$ of functions with the Baire property converges with respect to $\mathcal{J}$ to some real function $f$ with the Baire property $\left(f_{n} \underset{n \rightarrow \infty}{\stackrel{\mathcal{J}}{\longrightarrow}} f\right)$ if every subsequence $\left\{f_{m_{n}}\right\}_{n \in \mathbb{N}}$ of $\left\{f_{n}\right\}_{n \in \mathbb{N}}$ contains a subsubsequence $\left\{f_{m_{p_{n}}}\right\}_{n \in \mathbb{N}}$ which converges to $f$ J-a.e. (see [21]).

The point 0 is an J-density point of $A$ if

$$
\chi_{(n \cdot A) \cap(-1,1)} \underset{n \rightarrow \infty}{\stackrel{\mathcal{J}}{\longrightarrow}} \chi_{(-1,1)} .
$$

We say that $x$ is an J-density point of $A$ if 0 is an J-density point of $A-x=$ $\{a-x: a \in A\}$. Put $\Phi_{\mathcal{J}}(A)=\{x \in \mathbb{R}: x$ is an J-density point of $A\}$.

The family $\tau_{\mathcal{J}}=\left\{A \subset \mathbb{R}: A \in \mathcal{B} r \wedge A \subset \Phi_{\mathcal{J}}(A)\right\}$, called the J-density topology, was studied in [21], [22] and [25]. 
A set $A \subset \mathbb{R}$ is said to be semi-open if there is an open set $U$ such that $U \subset A \subset \bar{U}$ (see [15]). It is not difficult to see that $A$ is semi-open if and only if $A \subset \overline{\operatorname{Int}(A)}$. The family of all semi-open sets will be denoted by $\mathcal{S}$. A function $f$ is semi-continuous if for each open set $V$ the set $f^{-1}(V)$ is semi-open [15].

In [20], A. N e u b r u n o vá proved that $f$ is semi-continuous if and only if it is quasi-continuous.

Now, we are in the position to describe all the mentioned classes of functions using the notion of $\mathcal{A}$-continuity.

Remark 2. $\mathcal{A}$-continuity coincides with:

(1) classical continuity whenever $\mathcal{A}=\tau_{e}$;

(2) approximate continuity whenever $\mathcal{A}=\tau_{d}$;

(3) J-approximate continuity whenever $\mathcal{A}=\tau_{d_{J}}$;

(4) quasi-continuity whenever $\mathcal{A}=\mathcal{S}$.

Definition 3 ([10]). We will say that $f$ has the $\mathcal{A}$-Darboux property $\left(f \in \mathcal{D}_{\mathcal{A}}\right)$ if for each interval $(a, b) \subset \mathbb{R}$ and each $\lambda \in<f(a), f(b)>$ there exists a point $x \in(a, b)$ such that $f(x)=\lambda$ and $f$ is $\mathcal{A}$-continuous at $x$.

If $\mathcal{A}=\mathcal{P}(\mathbb{R})$ then $\mathcal{D}_{\mathcal{A}}$ is a family $\mathcal{D}$ of all Darboux functions. If $\mathcal{A}$ is the Euclidean topology $\tau_{e}$, then $\mathcal{D}_{\mathcal{A}}$ is a family $\mathcal{D}_{s}$ of functions having strong Świątkowski property. If $\mathcal{A}$ is the density topology $\tau_{d}$, then $\mathcal{D}_{\mathcal{A}}$ is a family $\mathcal{D}_{a p}$ of functions with the so called ap-Darboux property introduced by Z. G r a n d e in [6. If $\mathcal{A}$ is the J-density topology, then $\mathcal{D}_{\mathcal{A}}$ is a family of $\mathcal{J}$-ap Darboux functions investigated by G. Ivanova and E. Wagner-Bojakowska in [7] and $\left[9\right.$. In [10, it is shown that if $\mathcal{A}=\mathcal{S}$, then $\mathcal{D}_{\mathcal{A}}=\mathcal{D} \mathcal{Q}$.

It is easy to see that

$$
\mathcal{D}_{s} \subset \mathcal{D}_{a p} \cap \mathcal{D}_{\mathcal{J}_{-a p}} \subset \mathcal{D}_{a p} \cup \mathcal{D}_{\mathcal{J}_{-a p}} \subset \mathcal{D} .
$$

In [7, it is proved that all these inclusions are proper.

In [10, it is proved that for some families $\mathcal{A}$ we also have $\mathcal{D}_{\mathcal{A}} \subset \mathcal{D} Q$. Let us briefly describe these results. We say that the set $A$ is of the first category at the point $x$ if there exists an open neighbourhood $G$ of $x$ such that $A \cap G$ is of the first category (see [13]). $D(A)$ will denote the set of all points $x$ such that $A$ is not of the first category at $x$.

Definition 4 ([10]). We will say that a family $\mathcal{A} \subset \mathcal{P}(\mathbb{R})$ has the (*)-property, if

(1) $\tau_{e} \subset \mathcal{A} \subset \mathcal{B} r$

(2) $A \subset D(A)$ for each $A \in \mathcal{A}$.

It is not difficult to see that a wide class of topologies has the (*)-property. For example, the Euclidean topology, J-density topology, topologies constructed 
in 114 by E. Eazarow, R. A. Johnson and W. Wilczyński or the topology constructed by R. W i e r t e la k in [23]. Certain families of sets, which are not topologies, have the $(*)$-property: the family of semi-open sets is a good example. On the other hand, the density topology does not have this property.

\section{Algebrability}

To compare families $\mathcal{D}_{s}, \mathcal{D} \mathcal{Q}, \mathcal{D}_{a p}$ and $\mathcal{D}_{\mathcal{J}_{-a p}}$ in algebraic terms, we need some definitions.

Definition 5 ([3]). Let $\mathcal{L}$ be a linear commutative algebra. We say that $A \subset \mathcal{L}$ is strongly $\mathfrak{c}$-algebrable if $A \cup\{\Theta\}$ contains a $\mathfrak{c}$-generated algebra $B$ that is isomorphic with a free algebra. We denote by $X=\left\{x_{\alpha}: \alpha<\mathfrak{c}\right\}$ the set of generators of this free algebra.

Let us remark that the set $X=\left\{x_{\alpha}: \alpha<\mathfrak{c}\right\}$ is the set of generators of some free algebra contained in $A \cup\{\Theta\}$ if and only if the set $\widetilde{X}$ of elements of the form $x_{\alpha_{1}}^{k_{1}} x_{\alpha_{2}}^{k_{2}} \ldots x_{\alpha_{n}}^{k_{n}}$ is linearly independent and all linear combinations of elements from $\widetilde{X}$ are in $A \cup\{\Theta\}$.

In 2] there was presented a useful method of proving that a fixed family is strongly $\mathfrak{c}$-algebrable. We say that a function $f$ is exponential-like (briefly $f \in \mathcal{E}$ ) whenever $f$ is given by the formula

$$
f(x)=\sum_{i=1}^{m} a_{i} e^{\beta_{i} x},
$$

for some distinct nonzero real numbers $\beta_{1}, \ldots, \beta_{m}$ and some nonzero real numbers $a_{1}, \ldots, a_{m}$ (see [1]).

It is not difficult to check that:

LemMa 6 ([1]). For every positive integer $m$, any exponential-like function $f$, and each $c \in \mathbb{R}$, the preimage $f^{-1}[\{c\}]$ is finite. Consequently, $f$ is not constant in any subinterval of $\mathbb{R}$. In particular, there exists a decomposition of $\mathbb{R}$ to a finite number of intervals such that the function $f$ is strictly monotone on each of them.

Exponential-like functions may be used to prove $\mathfrak{c}$-algebrability of a fixed family of functions in the following way:

THEOREM 7 ([1]). Let $\mathcal{F} \subset \mathbb{R}^{\mathbb{R}}$ and assume that there exists a function $F \in \mathcal{F}$ such that $f \circ F \in \mathcal{F} \backslash\{\Theta\}$ for every exponential-like function $f$ [1]. Then, $\mathcal{F}$ is strongly $\mathfrak{c}$-algebrable. 


\section{Comparison of classes related to Darboux property}

In [8], it is shown that the family $\mathcal{D} \mathcal{Q}$ is strongly porous (so, it is very small from the topological point of view) in the family $\mathcal{D B} a$ of Darboux functions having the Baire property. Therefore, $\mathcal{D B} a \backslash \mathcal{D} \mathcal{Q}$ is "topologically large". Let us show that it is large also in algebraic terms. For this purpose, we need the following lemma:

LemMA 8 ([11]). There exists a Darboux Baire 1 function $F: \mathbb{R} \stackrel{\text { onto }}{\longrightarrow}[0,1]$ such that

(1) F vanishes J-a.e. on $(0,1)$;

(2) F vanishes on $\mathbb{R} \backslash(0,1)$;

(3) $[0,1] \backslash F^{-1}(\{0\})$ is a first category set dense in $[0,1]$.

Indeed, let $\left\{C_{n}\right\}$ be a sequence of pairwise disjoint closed and nowhere dense subsets of $[0,1]$ of cardinality continuum such that for each interval $(a, b) \subset[0,1]$ there exists $n \in \mathbb{N}$ with $C_{n} \subset(a, b)$. Obviously, the set $C=\bigcup_{n \in \mathbb{N}} C_{n}$ is of type $F_{\sigma}$ and is bilaterally $\mathfrak{c}$-dense-in-itself. Therefore, (see [4]) there exists a function $F \in \mathcal{D B}_{1}$ such that $F(x)=0$ if $x \notin C$ and $0<F(x) \leq 1$ for $x \in C$ (for details, see [11]).

Using this function, we can prove strong $\mathfrak{c}$-algebrability of the family $\mathcal{D B} a \backslash \mathcal{D Q}:$

TheOREM 9. The family $\mathcal{D B} a \backslash \mathcal{D} \mathcal{Q}$ is strongly $\mathfrak{c}$-algebrable.

P r o of. Let $F$ be the function defined above. Fix an exponential-like function $f$. By Theorem 7 to prove that $\mathcal{D B} a \backslash \mathcal{D} \mathcal{Q}$ is strongly c-algebrable, it is suffices to show that $f \circ F \in \mathcal{D B} a \backslash \mathcal{D} \mathcal{Q}$.

As $f$ is continuous and $F \in \mathcal{D B}_{1}, f \circ F \in \mathcal{D B} a$. To show that $f \circ F$ is not quasi-continuous, fix an open set $V$ such that $0 \notin f^{-1}(V)$ and $V \cap(f \circ F)(\mathbb{R}) \neq \emptyset$. The set $F^{-1}\left(f^{-1}(V)\right)$ is a nonempty set of the first category, so it is not semi-open, and due to A. Neubrunnová's result [20], the function $f \circ F$ is not quasi-continuous.

Put $\mathcal{P}:=\{\mathcal{D}, \mathcal{D} \mathcal{B} a\}$, and $\mathcal{P}_{\mathcal{A}}:=\left\{\mathcal{D}_{\mathcal{A}}: \mathcal{A}\right.$ has the $\left(^{*}\right)$-property $\}$. As for each $\mathcal{A}$ with the $(*)$-property, the family $\mathcal{D}_{\mathcal{A}}$ is contained in $\mathcal{D} \mathcal{Q}$ (see [10]), it is easy to see that for any $\mathcal{F}_{1} \in \mathcal{P}$ and $\mathcal{F}_{2} \in \mathcal{P}_{\mathcal{A}}$ we have $\mathcal{D B} a \subset \mathcal{F}_{1}$ and $\mathcal{F}_{2} \subset \mathcal{D} Q$. Consequently, by the latter theorem, we obtain

CoRollary 10. If $\mathcal{F}_{1} \in \mathcal{P}$ and $\mathcal{F}_{2} \in \mathcal{P}_{\mathcal{A}}$ then the family $\mathcal{F}_{1} \backslash \mathcal{F}_{2}$ is strongly c-algebrable. 


\section{MAŁGORZATA FILIPCZAK — GERTRUDA IVANOVA}

Remind that any family from $\mathcal{P}_{\mathcal{A}}$ is strongly porous in any family from $\mathcal{P}[8$. Therefore, if $\mathcal{F}_{1} \in \mathcal{P}$ and $\mathcal{F}_{2} \in \mathcal{P}_{\mathcal{A}}$, then $\mathcal{F}_{1} \backslash \mathcal{F}_{2}$ is residual (so, "topologically large") in $\mathcal{F}_{1}$.

Let us now consider some subfamilies of the family $\mathcal{B}_{1}$ of all Baire 1 functions. In [11, it is shown that a strong Świątkowski function need not be Baire 1, so no family from $\mathcal{P}_{\mathcal{A}}$ is contained in $\mathcal{B}_{1}$. Put $\mathcal{P}_{\mathcal{A}}^{\prime}:=\left\{\mathcal{D}_{\mathcal{A}} \cap \mathcal{B}_{1}: \mathcal{A}\right.$ has the (*)-property $\}$ and $\mathcal{D} \mathcal{B}_{1}$ - the family of all Darboux Baire 1 functions. In [11, it is proved that each family from $\mathcal{P}_{\mathcal{A}}^{\prime}$ is strongly porous, so "topologically small", in $\mathcal{D B}_{1}$. Using the function from Lemma 8, we can show, in the same way as in Theorem 9] that:

THEOREM 11. If $\mathcal{F} \in \mathcal{P}_{\mathcal{A}}^{\prime}$ then the family $\mathcal{D B}_{1} \backslash \mathcal{F}$ is strongly $\mathfrak{c}$-algebrable.

J. W o d k a proved in [27] that the family $\mathcal{D} Q \backslash \mathcal{D}_{s}$ is strongly c-algebrable. We will show that both $\left(\mathcal{C}_{\tau_{d}} \cap \mathcal{C}_{\tau_{\mathcal{J}}}\right) \backslash \mathcal{D}_{s}$ and $\mathcal{D}_{s} \backslash\left(\mathcal{C}_{\tau_{d}} \cup \mathcal{C}_{\tau_{\mathcal{J}}}\right)$ are strongly c-algebrable, too.

Recall that a set $A$ is called a right-interval set if $A$ is a union of intervals $\left(a_{n}, b_{n}\right)$ with $\lim _{n \rightarrow \infty} a_{n}=0$ and $0<b_{n+1}<a_{n}<b_{n}$ for each $n \in \mathbb{N}$. Suppose that $A=\bigcup_{n=1}^{\infty}\left(a_{n}, b_{n}\right)$ is a right-hand interval set and $b_{1}=1$.

Put

$$
t_{A}(x)= \begin{cases}1 & \text { for } x \leq 0, \\ 1-\frac{1}{n} & \text { for } x \in\left[a_{n}, b_{n}\right], n \in \mathbb{N} \\ 0 & \text { for } x=\frac{a_{n}+b_{n+1}}{2}, n \in \mathbb{N} \text { and for } x \in\left[b_{1}, \infty\right), \\ \text { linear } & \text { on the intervals }\left[b_{n+1}, \frac{a_{n}+b_{n+1}}{2}\right],\left[\frac{a_{n}+b_{n+1}}{2}, a_{n}\right], n \in \mathbb{N}\end{cases}
$$

and

$$
F_{A}(x)= \begin{cases}t_{A}(x-m)-m & \text { if } m \text { is even and } x \in[m, m+1], \\ 1-x & \text { if } m \text { is odd and } x \in(m, m+1) .\end{cases}
$$

Note that $F_{A}$ is not continuous at any even $m$, and $F_{A}$ is continuous at any other point. Moreover, $F_{A} \notin \mathcal{D}_{s}$.

LEMMA 12. Let $f$ be an exponential-like function. If $A$ is a right-hand interval set, then $f \circ F_{A} \notin \mathcal{D}_{s}$.

P r o of. Fix an exponential-like function $f$ and a right-hand interval set $A$. We will show that $f \circ F_{A}$ is not continuous and does not belong to $\mathcal{D}_{s}$. By Lemma [6] there exists an even number $m \in \mathbb{Z}$ such that $f$ is strictly monotone on $(-m,-m+2)$. Without loss of generality, we can assume that $f$ is strictly increasing on this interval.

Observe that $f \circ F_{A}$ is not continuous at $m$. Indeed, let $(a, b)$ be an arbitrary interval such that $m-1<a<m<b<m+1$. Then

$$
f \circ F_{A}((a, b)) \supset f([-m,-m+1]) .
$$


Since $f$ is strictly monotone and continuous on $[-m,-m+1], f([-m$, $-m+1])$ is a nondegenerate interval. Let $\epsilon<\operatorname{diam}(f([-m,-m+1])) / 3$. Then,

$$
\left(f\left(F_{A}(m)\right)-\epsilon, f\left(F_{A}(m)\right)+\epsilon\right) \nsupseteq f\left(F_{A}(a, b)\right),
$$

for each $(a, b)$ such that $m-1<a<m<b<m+1$.

Let us show that $f \circ F_{A} \notin \mathcal{D}_{s}$. For this purpose, fix numbers $a_{0}$ and $b_{0}$ such that $m-1<a_{0}<m<b_{0}<m+1$. Then, we have $F_{A}\left(a_{0}\right)>F_{A}(m)>F_{A}\left(b_{0}\right)$ and $F_{A}(m)=-m+1$. As $f$ is strictly increasing on $(-m,-m+2)$, we obtain $f\left(F_{A}\left(a_{0}\right)\right)>f\left(F_{A}(x)\right)>f\left(F_{A}(m)\right)>f\left(F_{A}(y)\right)>f\left(F_{A}\left(b_{0}\right)\right)$ for all $x \in\left(a_{0}, m\right)$ and $y \in\left(m, b_{0}\right)$, so on the interval $\left(a_{0}, b_{0}\right)$ the function $f \circ F_{A}$ has value $f(-m+1)$ only at the point $m$. Note that $f \circ F_{A}$ is not continuous at $m$. Therefore, $f \circ F_{A}$ is not a strong Swiątkowski function.

Fix $f \in \mathcal{C}_{\tau_{d}}$. It is well-known [26] that $f$ has the Darboux property. Since $f$ is approximately continuous at any point, $f \in \mathcal{D}_{a p}$. Analogously, from the fact that $\mathrm{C}_{\tau_{d}} \subset \mathcal{D}$ [21, we obtain that $\mathcal{C}_{\tau_{\mathcal{J}}} \subset \mathcal{D}_{\mathcal{J}-a p}$.

As $\tau_{\mathcal{J}}$ has the $\left(^{*}\right)$-property, $\mathcal{D}_{\mathcal{J}-a p} \subset \mathcal{D} \mathcal{Q}$. Thus,

$$
\mathcal{C}_{\tau_{d}} \cap \mathcal{C}_{\tau_{\mathcal{J}}} \subset \mathcal{D}_{a p} \cap \mathcal{D}_{\mathcal{J}-a p} \subset \mathcal{D} \mathcal{Q} .
$$

Observe that families $\mathcal{C}_{\tau_{d}} \cap \mathcal{C}_{\tau_{\mathcal{J}}}$ and $\mathcal{D}_{s}$ are incomparable.

EXAMPLE 13. If 0 is a right-hand density point and a right-hand J-density point of a right-hand interval set $A$, then $t_{A} \in\left(\mathcal{C}_{\tau_{d}} \cap \mathcal{C}_{\tau_{\mathcal{J}}}\right) \backslash \mathcal{D}_{s}$.

Proof. Indeed, $t_{A}$ is continuous at any point $x \neq 0$. Moreover, $t_{A}$ is approximately continuous and J-approximately continuous at zero. On the other hand, $1 \in t_{A}((-1 / 2,1 / 2))$ and the only point $x_{0}$ such that $t_{A}\left(x_{0}\right)=1$ is equal to 0 , and $t_{A}$ is not continuous at zero.

ExAmple 14. There exists a function belonging to $\mathcal{D}_{s} \backslash\left(\mathcal{C}_{\tau_{d}} \cup \mathcal{C}_{\tau_{\jmath}}\right)$.

P r o of. Suppose that $A$ is a right-hand interval set at 0 such that 0 is a right-hand density point and J-density point of $A$. Put

$$
\hat{t}_{A}(x)= \begin{cases}1 & \text { for } x=\frac{a_{n}+b_{n+1}}{2}, n \in \mathbb{N}, \text { and for } x \leq 0, \\ 0 & \text { for } x \in\left[a_{n}, b_{n}\right], n \in \mathbb{N}, \text { and for } x \in\left(b_{1}, \infty\right), \\ \text { linear } & \text { on intervals }\left[b_{n+1}, \frac{a_{n}+b_{n+1}}{2}\right],\left[\frac{a_{n}+b_{n+1}}{2}, a_{n}\right], n \in \mathbb{N}\end{cases}
$$

and

$$
\hat{F}_{A}(x)= \begin{cases}\hat{t}_{A}(x-m)-m & \text { if } m \text { is even and } x \in[m, m+1], \\ 1-x & \text { if } m \text { is odd and } x \in(m, m+1) .\end{cases}
$$

Then, $\hat{F}_{A}$ is neither approximately nor J-approximately continuous at each $m \in \mathbb{Z}$ and has the strong Świątkowski property, so $\hat{F}_{A} \in \mathcal{D}_{s} \backslash\left(\mathcal{C}_{\tau_{d}} \cup \mathcal{C}_{\tau_{\mathcal{J}}}\right)$. 


\section{MAŁGORZATA FILIPCZAK — GERTRUDA IVANOVA}

Using functions $F_{A}$ and $\hat{F}_{A}$ considered in Example 13 and Example 14 we obtain that families $\left(\mathcal{C}_{\tau_{d}} \cap \mathcal{C}_{\tau_{\mathcal{J}}}\right) \backslash \mathcal{D}_{s}$ and $\mathcal{D}_{s} \backslash\left(\mathcal{C}_{\tau_{d}} \cup \mathcal{C}_{\tau_{\mathcal{J}}}\right)$ are strongly c-algebrable. Indeed, using the exponential method, we obtain the following facts:

THEOREM 15. The family $\left(\mathcal{C}_{\tau_{d}} \cap \mathcal{C}_{\tau_{\mathcal{J}}}\right) \backslash \mathcal{D}_{s}$ is strongly $\mathfrak{c}$-algebrable.

P r o of. Suppose that $A$ is a right-hand interval set such that $b_{1}=1$ and 0 is right-hand density and J-density point of $A$. Let $f$ be an exponential-like function. It is easy to check that $f \circ F_{A}$ is approximately and J-approximately continuous. By Lemma 12 , we obtain $f \circ F_{A} \in\left(\mathcal{C}_{\tau_{d}} \cap \mathcal{C}_{\tau_{\mathcal{J}}}\right) \backslash \mathcal{D}_{s}$.

Corollary 16. Let

$$
\begin{array}{r}
\mathcal{P}=\left\{\mathcal{D} Q, \mathcal{D}_{\mathcal{J}-a p}, \mathcal{D}_{a p}, \mathcal{D}_{a p} \cap \mathcal{D}_{\mathcal{J}-a p}, \mathcal{C}_{\tau_{d}}, \mathcal{C}_{\tau_{d}} \cup \mathcal{D}_{s}, \mathcal{C}_{\tau_{\mathcal{J}}}, \mathcal{C}_{\tau_{\mathcal{J}}} \cup \mathcal{D}_{s}, \mathcal{C}_{\tau_{d}} \cap \mathcal{C}_{\tau_{\mathcal{J}}},\right. \\
\left.\left(\mathcal{C}_{\tau_{d}} \cap \mathcal{C}_{\tau_{\mathcal{J}}}\right) \cup \mathcal{D}_{s}\right\} .
\end{array}
$$

Each $\mathcal{F} \in \mathcal{P}$ contains $\mathcal{C}_{\tau_{d}} \cap \mathcal{C}_{\tau_{\mathcal{J}}}$. Therefore, by the latter theorem, the family $\mathcal{F} \backslash \mathcal{D}_{s}$ is strongly $\mathfrak{c}$-algebrable for any $\mathcal{F}$ belonging to $\mathcal{P}$.

THEOREM 17. The family $\mathcal{D}_{s} \backslash\left(\mathcal{C}_{\tau_{d}} \cup \mathcal{C}_{\tau_{\mathcal{J}}}\right)$ is strongly $\mathfrak{c}$-algebrable.

P r oof. Fix an exponential-like function $f$, and let $\hat{F}_{A}$ be the function from Example 14. Let us show that $f \circ \hat{F}_{A} \in \mathcal{D}_{s}$. Indeed, fix $(a, b) \subset \mathbb{R}$ such that $f\left(\hat{F}_{A}(a)\right) \neq f\left(\hat{F}_{A}(b)\right)$, and $\lambda \in<f\left(\hat{F}_{A}(a)\right), f\left(\hat{F}_{A}(b)\right)>$. Then, as $f \circ \hat{F}_{A}$ has the Darboux property, there exists a point $x^{\prime} \in(a, b)$ such that $f \circ \hat{F}_{A}\left(x^{\prime}\right)=\lambda$.

If $x^{\prime} \notin \mathbb{Z}$ or $x^{\prime}$ is odd, then put $x=x^{\prime}$. It is easy to see that $f \circ \hat{F}_{A}$ is continuous at $x$.

Assume that there exists even $m$ such that $x^{\prime}=m$. Let us show that there exists a point $x \in\left(x^{\prime}, b\right)$ such that $f \circ \hat{F}_{A}(x)=\lambda$. Indeed, we can find a point $x \in\left(x^{\prime}, b\right)$ with $\hat{F}_{A}(x)=1-m$, so $f\left(\hat{F}_{A}(x)\right)=f\left(\hat{F}_{A}(m)\right)=\lambda$.

It is easy to see that $f \circ \hat{F}_{A}$ is continuous at $x$, so it has the strong Swiątkowski property.

We will show that $f \circ \hat{F}_{A}$ is neither approximately nor J-approximately continuous. By Lemma 6] there exists an even number $m \in \mathbb{Z}$ such that $f$ is strictly monotone on $[-m,-m+1]$. Without loss of generality, we can assume that $f$ is strictly increasing function on this interval, so $f(-m)<f(1-m)$. Let $\epsilon \in(0, f(1-m)-f(-m))$. Then,

$$
A+m \subset \mathbb{R} \backslash\left[\hat{F}_{A}^{-1}\left(f^{-1}\left(\left(f\left(\hat{F}_{A}(m)\right)-\epsilon, f\left(\hat{F}_{A}(m)\right)+\epsilon\right)\right)\right)\right],
$$

so $m$ is a right-hand dispersion and a right-hand J-dispersion point of $A+m$, and $f \circ \hat{F}_{A}$ is neither approximately nor J-approximately continuous at $m$.

THEOREM 18. The family $\mathcal{D} Q \backslash\left(\mathcal{D}_{a p} \cup \mathcal{D}_{\mathcal{J}-a p}\right)$ is strongly $\mathfrak{c}$-algebrable. 
P r o of. Let $B=\bigcup_{n=1}^{\infty}\left(a_{n}, b_{n}\right)$ be a right-hand interval set such that 0 is a density and an J-density point of the set $(-1,0] \cup B$.

We will use a function $f_{B}$ which is a modification of $t_{B}$.

Let

$$
f_{B}(x)= \begin{cases}1 & \text { for } x \leq 0, \\ 0 & \text { for } x \in\left[a_{n}, b_{n}\right], n \in \mathbb{N}, \text { and for } x \in\left(b_{1}, \infty\right), \\ 1-\frac{1}{n} & \text { for } x=\frac{a_{n}+b_{n+1}}{2}, n \in \mathbb{N}, \\ \text { linear } & \text { on intervals }\left[b_{n+1}, \frac{a_{n}+b_{n+1}}{2}\right],\left[\frac{a_{n}+b_{n+1}}{2}, a_{n}\right], n \in \mathbb{N} .\end{cases}
$$

Now, we use the function

$$
F_{B}(x)= \begin{cases}f_{B}(x-m)-m, & \text { if } m \text { is even and } x \in[m, m+1], \\ 1-x & \text { if } m \text { is odd and } x \in(m, m+1) .\end{cases}
$$

It is not difficult to see that $F_{B} \in \mathcal{D} \mathcal{Q}$.

Fix an exponential-like function $f$. Then, as $f$ is continuous, $f \circ F_{B} \in \mathcal{D} \mathcal{Q}$. There exists an even number $m \in \mathbb{Z}$ such that $f$ is strictly monotone on $(-m,-m+2)$. Again, we can assume that $f$ is strictly increasing on this interval.

Let $y_{0}=f(-m)$ and $y_{1}=f \circ F_{B}(m)=f(-m+1)$. Observe that $F_{B}(B)=\{0\}$, so $f\left(F_{B}(B+m)\right)=\left\{y_{0}\right\}$ and $y_{0}<y_{1}$.

Fix $\epsilon \in\left(0, y_{1}-y_{0}\right)$ and put $W=\left(y_{1}-\epsilon, y_{1}+\epsilon\right)$. The complement of the set $F_{B}^{-1}\left(f^{-1}(W)\right)$ contains the set $B+m$. As $f\left(F_{B}(m)\right)=y_{1}$ and $m$ is a right-hand density and a right-hand J-density point of $B+m, f \circ F_{B}$ is neither approximately nor J-approximately continuous at $m$. Therefore, as $f \circ F_{B}$ assumes value $-m$ on the interval $(m-1, m+1)$ only at the point $m$, we obtain $f \circ F_{B} \in \mathcal{D} \mathcal{Q} \backslash\left(\mathcal{D}_{a p} \cup \mathcal{D}_{\mathcal{J}-a p}\right)$.

Corollary 19. Put $\mathcal{P}=\left\{\mathcal{D}_{\mathcal{J}_{-a p}}, \mathcal{D}_{a p}, \mathcal{D}_{a p} \cup \mathcal{D}_{\mathcal{J}-a p}, \mathcal{C}_{\tau_{d}}, \mathcal{C}_{\tau_{\mathcal{J}}}, \mathcal{C}_{\tau_{d}} \cup \mathcal{C}_{\tau_{\mathcal{J}}}\right\}$. If $\mathcal{F} \in \mathcal{P}$, then the family $\mathcal{D} \mathcal{Q} \backslash \mathcal{F}$ is strongly $\mathfrak{c}$-algebrable.

\section{REFERENCES}

[1] BALCERZAK, M.-BARTOSZEWICZ, A.-FILIPCZAK, M.: Nonseparable spaceability and strong algebrability of sets of continuous singular functions, J. Math. Anal. Appl. 407 (2013), 263-269.

[2] BARTOSZEWICZ, A.-BIENIAS, M.-FILIPCZAK, M.-GŁĄB, S.: Strong c-algebrability of strong Sierpiński-Zygmunt, smooth nowhere analytic and other sets of functions, J. Math Anal. Appl. 412 (2014), 620-630.

[3] BARTOSZEWICZ, A.-GEĄB, S.: Strong algebrability of sets of sequences and functions, Proc. Amer. Math. Soc. 141 (2013), 827-835. 


\section{MAŁGORZATA FILIPCZAK — GERTRUDA IVANOVA}

[4] BRUCKNER, A. M.: Differentiation of Real Functions, in: Lecture Notes in Math., Vol. 659, Springer, Berlin, 1978.

[5] FILIPCZAK, M.-IVANOVA, G.-WÓDKA, J.: Comparison of some families of real functions in porosity terms, Math. Slovaca (to appear).

[6] GRANDE, Z.: On a subclass of the family of Darboux functions, Colloq. Math. 117 (2009), 95-104.

[7] IVANOVA, G.: Remarks on some modification of the Darboux property, Bull. Soc. Sci. Lett. Łódź Sér. Rech. Déform. 72 (2014), 91-100.

[8] IVANOVA, G.-KARASIŃSKA, A.-WAGNER-BOJAKOWSKA, E.: Comparison of some subfamilies of functions having the Baire property, Tatra Mt. Math. Publ. 65 (2016), 151-159.

[9] IVANOVA, G.-WAGNER-BOJAKOWSKA, E.: On some modification of Darboux property, Math. Slovaca 66 (2016), 79-88.

[10] IVANOVA, G.-WAGNER-BOJAKOWSKA, E.: On some modification of Świgtkowski property, Tatra Mt. Math. Publ. 58 (2014), 101-109.

[11] IVANOVA, G.-WAGNER-BOJAKOWSKA, E.: On some subclasses of the family of Darboux Baire 1 functions, Opuscula Math. 34 (2014), 777-788.

[12] KEMPISTY, S.: Sur les fonctions quasicontinues, Fund. Math. 19 (1932), 184-197.

[13] KURATOWSKI, A.-MOSTOWSKI, A.: Set Theory with an Introduction to Descriptive Set Theory. Warszawa, PWN, 1976.

[14] EAZAROW, E.-JOHNSON, R. A.-WILCZYŃSKI, W.: Topologies related to sets having the Baire property, Demonstratio Math. 21 (1989), 179-191.

[15] LEVINE, N.: Semi-open sets and semi-continuity in topological spaces, Amer. Math. Monthly 70 (1963), 36-41.

[16] MALISZEWSKI, A.: Darboux Property and Quasi-Continuity: A Uniform Approach. WSP, Słupsk, 1996.

[17] MALISZEWSKI, A.: On the limits of strong Światkowski functions, Zeszyty Nauk. Politech. Łódz. Mat. 27 (1995), 87-93.

[18] MALISZEWSKI, A.: On the averages of Darboux functions, Trans. Amer. Math. Soc. 350 (1998), 2833-2846.

[19] MALISZEWSKI, A.: Sums and products of quasi-continuous functions, Real Anal. Exchange 21 (1995/1996), 320-329.

[20] NEUBRUNNOVÁ, A.: On certain generalizations of the notion of continuity, Matematický časopis 23 (1973), 374-380.

[21] POREDA, W.-WAGNER-BOJAKOWSKA, E.-WILCZYŃSKI, W.: A category analogue of the density topology, Fund. Math. 125 (1985), 167-173.

[22] POREDA, W.-WAGNER-BOJAKOWSKA,E.-WILCZYŃSKI, W.: Remarks on J-density and J-approximately continuous functions, Comm. Math. Univ. Carolinae 26 (1985), $553-563$.

[23] WIERTELAK, R.: A generalization of density topology with respect to category, Real Anal. Exchange 32 (2006/2007), 273-286.

[24] WILCZYŃSKI, W.: A generalization of the density topology, Real Anal. Exchange 8 (1982/1983), 16-20. 


\title{
COMPARISON OF SOME FAMILIES OF REAL FUNCTIONS IN ALGEBRAIC TERMS
}

[25] WILCZYŃSKI, W.: A category analogue of the density topology, approximate continuity and the approximate derivative, Real Anal. Exchange 10 (1984/1985), 241-265.

[26] WILCZYŃSKI, W.: Density topologies, in: Handbook of Measure Theory, Vol. I and II (E. Pap, ed.), North-Holland, Amsterdam, 2002, pp. 675-702.

[27] WÓDKA, J.: Subsets of some families of real functions and their algebrability, Linear Algebra Appl. 459 (2014), 454-464.

Received November 19, 2015

\author{
Matgorzata Filipczak \\ Faculty of Mathematics and \\ Computer Sciences \\ Eódź University \\ Stefana Banacha 22 \\ $P L-90-238$ Eódź \\ POLAND \\ E-mail: malfil@math.uni.lodz.pl \\ Gertruda Ivanova \\ Institute of Mathematics \\ Pomeranien University in Stupsk \\ Kozietulskiego 6-7 \\ PL-76-200 Stupsk \\ POLAND \\ E-mail: gertruda@apsl.edu.pl
}

